\title{
Social anxiety disorder in first-episode psychosis: incidence, phenomenology and relationship with paranoia
}

\author{
Maria Michail and Max Birchwood
}

\section{Background}

Social anxiety disorder constitutes a significant problem for people with psychosis. It is unclear whether this is a by-product of persecutory thinking.

\section{Aims}

To compare the phenomenology of social anxiety disorder in first-episode psychosis with that in a group without psychosis. The relationship between social anxiety and psychosis symptoms was investigated.

\section{Method}

A sample of people with first-episode psychosis (FEP group) was compared with a sample with social anxiety disorder without psychosis (SaD group).

\section{Results}

Of the individuals in the FEP group $(n=80) 25 \%$ were diagnosed with an ICD-10 social anxiety disorder (FEP/SaD group); a further $11.6 \%$ reported severe difficulties in social encounters. The FEP/SaD and SaD groups reported comparable levels of social anxiety, autonomic symptoms, avoidance and depression. Social anxiety in psychosis was not related to the positive symptoms of the Positive and Negative Syndrome Scale (PANSS) including suspiciousness/ persecution. However, a significantly greater percentage of socially anxious $v$. non-socially anxious individuals with psychosis expressed perceived threat from persecutors, although this did not affect the severity of social anxiety within the FEP/SaD group. The majority of those in the FEP/ SaD group did not have concurrent persecutory delusions.

\section{Conclusions}

Social anxiety is a significant comorbidity in first-episode psychosis. It is not simply an epiphenomenon of psychotic symptoms and clinical paranoia, and it has more than one causal pathway. For a subgroup of socially anxious people with psychosis, anticipated harm is present and the processes that underlie its relationship with social anxiety warrant further investigation.

\section{Declaration of interest}

None.
Factor analytic studies of psychosis symptoms consistently point to a depression dimension in non-affective psychosis. ${ }^{1}$ Depression and social anxiety are each observed throughout the course, ${ }^{2}$ including the prodromal phase ${ }^{3,4}$ and following symptomatic recovery. Post-psychotic depression has been reported in 30$50 \%$ of individuals ${ }^{5,6}$ and social anxiety disorder has been observed in up to one in three..$^{7-10}$ In the first year following a first episode of psychosis, a rate of $29 \%$ with social anxiety disorder has been reported. ${ }^{11}$ The nature and origin of social anxiety disorder in psychosis is not well understood; when present, however, it exerts a significant impact on social disability. ${ }^{8,10,12,13}$ Social anxiety without psychosis tends to go hand-in-hand with depression; they are correlated cross-sectionally and, as it develops during adolescence, social anxiety usually precedes the onset of depression, ${ }^{14}$ suggesting the operation of overlapping mechanisms. However, in enduring psychosis, Pallanti et $a l^{15}$ report no link between social anxiety disorder and lifetime depression; indeed they argue that social anxiety disorder in psychosis is qualitatively different from 'non-psychotic' social anxiety disorder, and essentially a 'phenocopy'. They propose that non-psychotic social anxiety disorder is closer to depression ${ }^{15}$ with more autonomic anxiety symptoms, whereas social anxiety disorder in psychosis is atypical and a manifestation of psychotic symptoms (e.g. 'selfreferential attitude'), including the 'basic symptoms' and cognitive deficit, each impairing social cognition. This view of social anxiety casts it as a developmental 'endophenotype' of schizophrenia, and is consistent with the Edinburgh High Risk Study ${ }^{4,16}$ that found 'situational' anxiety to be common in their high-risk sample and among the best predictors of transition to psychosis, though diminishing when psychosis was manifest.

The relationship between anxiety and psychosis has attracted considerable attention in the literature. Freeman et al, ${ }^{17}$ examining the role of anxiety in the development of persecutory delusions, propose that similar themes and processes underlie both. Anxiety is a defensive reaction to the anticipation of threat and danger (physical, social or psychological); persecutory delusions are characterised by similar themes referring to perceived danger or harm from another. Freeman et $\mathrm{al}^{17}$ argue that anxiety is inherent in paranoia and is likely to play an important role in the formation and maintenance of persecutory delusions.

The aim of this study is to determine whether social anxiety disorder in psychosis is qualitatively different from social anxiety disorder in patients without psychosis. We compare social anxiety disorder in psychosis with an age-matched social anxiety disorder group without psychosis, to address the following questions: is the phenomenology of social anxiety disorder with psychosis different from that of social anxiety disorder without psychosis, including the presence of comorbid depression and autonomic symptoms; and is social anxiety disorder with psychosis linked to the nature or severity of psychosis symptoms and the perceived threat from persecutors?

We studied people presenting with a first episode of psychosis within 24 months of onset to reduce the possibility that social anxiety disorder and persecutory thinking become mutually contaminated with time, and in order to be able to study processes proximal to the early development of social anxiety disorder. 


\section{Method}

\section{Participants}

Inclusion criteria

Three groups of participants between 16 and 35 years were sampled: a group with social anxiety disorder without psychosis $(\mathrm{SaD}$ group); a group with first-episode psychosis (FEP group); and healthy controls (not reported here). Participants were assessed using the Schedules for Clinical Assessment in Neuropsychiatry $(\mathrm{SCAN})^{18}$ by M.M. who received formal training to criterion levels of reliability (M.M attended a 5-day training course on SCAN at the University of Leicester. Reliability monitoring was conducted between SCAN trainees and trainers. Positive and Negative Syndrome Scale (PANSS) ${ }^{19}$ training was provided at the Early Intervention Service, Birmingham and Solihull Mental Health Foundation Trust). Individuals in the $\mathrm{SaD}$ group were required to conform to ICD-10 criteria $^{20}$ (F40.1) for social anxiety disorder; and in the FEP group, with ICD-10 criteria for schizophrenia or related disorder (F20, 22, 23), in the absence of a primary diagnosis of organic disorders.

\section{Recruitment}

Participants with first-episode psychosis were recruited from consecutive cases managed in the Early Intervention Service of Birmingham and Solihull Mental Health NHS Foundation Trust, UK. The service manages all individuals with first-episode psychosis in those aged 14-35 years, in Birmingham (population 1.2 million). People with social anxiety were recruited via a selfhelp organisation, Social Anxiety UK (www.social-anxiety.org.uk). People with social anxiety were also approached via local community mental health teams. However, recruitment proved challenging as individuals were reluctant to engage in face-to-face interviews.

\section{Procedure}

The recruitment procedure involved the researcher (M.M.) contacting each potential participant individually to inform them about the purpose of the study and to invite them to take part. A signed consent form was obtained from those agreeing to participate. Testing consisted of a clinical interview using the SCAN for the ICD-10 criteria in order to establish diagnoses of social anxiety disorder and/or schizophrenia. The SCAN permits enquiry about the history, duration and impact of psychopathology in adults. Upon completion of the interview, data are entered in specialised software (CATEGO; IShell for SCAN, release 1, ed. 1.0.3.5, Win 9xNT, World Health Organization) which provides the diagnosis. Following diagnostic screening, an interview was conducted using the measures outlined below.

\section{Assessments}

Social anxiety

Three scales widely used together in the social phobia literature were administered. The Social Interaction Anxiety Scale (SIAS) ${ }^{21}$ is a 20 -item scale measuring anxiety in interpersonal encounters. Using a cut-off score of 36 , the SIAS has been demonstrated to discriminate between social anxiety, other anxiety disorders and community samples, ${ }^{22}$ with a sensitivity of 0.93 and a positive predictive value of 0.84 .

The Social Phobia Scale (SPS), ${ }^{21}$ designed to be administered alongside the SIAS, is used to detect and assess performance anxiety in situations where the individual fears they are being observed and scrutinised by others (e.g. carrying a tray across a cafeteria, eating/drinking in public).
The Brief Fear of Negative Evaluation Scale $(\mathrm{B}-\mathrm{FNE})^{23}$ is a 12-item self-report scale assessing concerns about negative evaluation by others in social encounters. Used extensively in social phobia research, it assesses evaluative cognitions believed to maintain social phobia. ${ }^{24}$

\section{Psychosis}

The Positive and Negative Syndrome Scale (PANSS) ${ }^{19}$ includes scales of positive symptoms, negative symptoms and general psychopathology, and is used widely in schizophrenia research.

The Details of Threat Questionnaire (DoT) $)^{25}$ was used to assess the nature of the perceived threat arising from persecutory delusions including the dimensions of: the power of persecutor (0-10 scale), the strength of delusional conviction $(0-100 \%)$, the perceived impact or 'awfulness' of threat (0-10 scale) and perceived controllability of the threat $(0-10$ scale). All participants were initially asked a screening question ('Do you think that anyone is trying to harm you in any way?') to ascertain the presence/absence of persecutory threat.

\section{Depression}

The Calgary Depression Scale for Schizophrenia (CDSS) ${ }^{26}$ is a nine-item observer-rated measure specifically designed for schizophrenia, minimising contamination by negative symptoms and the extrapyramidal side-effects of antipsychotics. It is strongly correlated with the Beck Depression Inventory (BDI) $)^{27}(r=0.91)$ and is responsive to change in psychosis. ${ }^{28}$ Recent studies have also supported the use of the CDSS in a healthy population without psychosis. $^{29}$

\section{Results}

\section{Participants}

Eighty-four people with first-episode psychosis were approached to take part in the study of whom eighty (95.2\%) consented. Following SCAN assessment the FEP group was divided into those with (FEP/SaD) and without (FEP/no SaD) social anxiety. An agematched healthy control group $(n=24)$ was also recruited (but not reported here). Table 1 presents information on the demographic characteristics of the samples. Participants in the $\mathrm{SaD}$ group were approximately 3 years older than those in the FEP groups. Chi-squared tests showed significant differences between the three groups in ethnicity $\left(\chi_{(8)}^{2}=52.9, P<0.01\right)$, education $\left(\chi_{(6)}^{2}=16.7, P<0.05\right)$ and occupation $\left(\chi_{(6)}^{2}=14.4, P<0.05\right)$, but not in marital status $\left(\chi_{(6)}^{2}=8.6\right.$, not significant). These differences were accounted for by the $\mathrm{SaD}$ group, who were more likely to have a higher level of education and to be in employment. There was a significant gender difference between the groups $\left(\chi_{(2)}^{2}=19.3, P<0.01\right)$; a female excess in both the $\mathrm{SaD}$ and $\mathrm{FEP} /$ no SaD groups showed the expected male excess.

\section{Prevalence of social anxiety in first-episode psychosis}

Overall, 20 (25\%) out of 80 people with first-episode psychosis received an ICD-10 diagnosis of social anxiety disorder based on the SCAN. All twenty scored above the cut-off points on both the SIAS $(>36)$ and the SPS (>26). Premorbid levels of social anxiety were available using the SCAN; participants were asked about the presence and severity of panic attacks, generalised anxiety and phobias (section 4 of SCAN) before and after the onset of psychosis. Data were entered in CATEGO which, after processing it, provides an ICD-10 (and DSM-IV) ${ }^{30}$ diagnosis for current and premorbid status. Out of the 20 people in the 


\begin{tabular}{|c|c|c|c|c|}
\hline & $\operatorname{FEP}(n=60)$ & $\mathrm{FEP} / \mathrm{SaD}(n=20)$ & $\operatorname{SaD}(n=31)$ & $P$ \\
\hline Gender, $n$ & & & & $<0.01$ \\
\hline Male & 46 & 7 & 11 & \\
\hline Female & 14 & 13 & 20 & \\
\hline Age, years: mean (s.d.) & $24.6(4.5)$ & $24.4(5.1)$ & $27.6(5)$ & $<0.05$ \\
\hline Ethnic origin, $n$ & & & & $<0.01$ \\
\hline African-Caribbean & 9 & 2 & 0 & \\
\hline Asian & 30 & 8 & 1 & \\
\hline British - White & 11 & 7 & 29 & \\
\hline British - Black & 10 & 2 & 1 & \\
\hline Other & 0 & 1 & 0 & \\
\hline Education, ${ }^{a} n$ & & & & $<0.05$ \\
\hline Left school & 27 & 5 & 2 & \\
\hline GSCE & 9 & 5 & 8 & \\
\hline A levels & 17 & 7 & 12 & \\
\hline Degree/HND & 7 & 2 & 9 & \\
\hline Occupation, $n$ & & & & $<0.05$ \\
\hline Employed & 10 & 4 & 15 & \\
\hline Unemployed & 41 & 12 & 10 & \\
\hline Student & 8 & 3 & 4 & \\
\hline Household & 1 & 1 & 2 & \\
\hline Marital status, $n$ & & & & NS \\
\hline Single & 50 & 17 & 20 & \\
\hline Cohabiting & 3 & 1 & 5 & \\
\hline Married & 6 & 1 & 6 & \\
\hline Separated & 1 & 1 & 0 & \\
\hline
\end{tabular}

$\mathrm{FEP} / \mathrm{SaD}$ group, 12 were able to provide detailed relevant information. None of these 12 were classified by CATEGO with premorbid social anxiety. These participants responded negative to the screening questions about experiencing feelings of anxiety, panic attacks and phobias before the onset of their illness. When prompted further about specific anxiety symptoms, they all reported with certainty that these became prominent following their first episode.

Of the remaining participants in the FEP/no SaD group, seven $(11.6 \%)$ nevertheless scored above the cut-off point on either the SIAS or SPS or both scales, despite not receiving a formal SCAN diagnosis of social anxiety. These individuals reported during interview clear difficulties in social interaction or signs of avoidance, but not sufficient to reach diagnostic criteria for social anxiety. A closer look at the SIAS and SPS scores of individuals in this group revealed that aside from these borderline cases that exceeded the cut-off points, there were also people $(n=8)$ scoring very close to the margins (30-36 on the SIAS and 20-26 on the SPS). This suggests that these people experience some social difficulties probably of moderate or 'subsyndromal' levels.

\section{Social anxiety in the with $\boldsymbol{v}$. without psychosis group}

In order to examine the extent to which the $\mathrm{SaD}$ and FEP/SaD groups share similar or different psychopathological features, we tested for differences in the severity of: social anxiety symptomatology; autonomic symptoms of anxiety; fear of negative social evaluation; and depression. To examine for differences in severity of autonomic symptoms, we used the list of autonomic symptoms in SCAN section 4 as a separate scale (16 symptoms rated as: absent, 0 ; symptoms present at different times, 1; symptoms present and occurring together e.g. during a panic attack, 2; and both situations present, 3). A reliability analysis found Cronbach's alpha for this scale to be 0.91 ; we therefore summed scores to obtain the total number of autonomic symptoms of anxiety.
Examining for differences in the prevalence of autonomic symptoms of anxiety reported by individuals in the $\mathrm{SaD}$ and $\mathrm{FEP} / \mathrm{SaD}$ groups, a one-way ANOVA with the total number of SCAN autonomic symptoms as a dependent variable revealed no significant differences between the two socially anxious groups (with $v$. without psychosis) $\left(F_{(1,48)}=2.46\right.$, not significant).

A one-way between-participants ANOVA comparing the FEP/ $\mathrm{SaD}$ and $\mathrm{SaD}$ groups, revealed no significant differences in the SIAS $\left(F_{(1,49)}=2.55\right.$ not significant $)$ or SPS $\left(F_{(1,49)}=1.65\right.$, not significant). However, the $\mathrm{SaD}$ group scored significantly higher on the B-FNE scale $\left(F_{(1,48)}=12.69, P<0.01\right)$ (Table 2). There was a significant difference in depression (CDSS) between the groups $\left(F_{(1,49)}=0.26\right.$, not significant), with $64.5 \%$ of the $\mathrm{SaD}$ and $65 \%$ of the $\mathrm{FEP} / \mathrm{SaD}$ groups shown to be at least moderately depressed.

In summary, the severity of social anxiety, including specific autonomic anxiety symptoms, social avoidance and depression in people with social anxiety disorder alone is comparable with that of people with psychosis and social anxiety disorder. Fear of negative evaluation was somewhat higher in the $\mathrm{SaD}$ group.

\section{Presence of other anxiety disorders}

Table 3 shows the prevalence of other ICD-10 anxiety disorders in the FEP (with and without social anxiety) and the $\mathrm{SaD}$ (without psychosis) groups. Apart from social anxiety (25\%), a significant proportion of individuals in the FEP group (16.2\%) were diagnosed with a comorbid anxiety disorder, the most prominent being panic disorder (10\%).

We were interested to determine whether social anxiety in both $\mathrm{SaD}$ and $\mathrm{FEP} / \mathrm{SaD}$ groups occurred in the context of a similar rate of other ICD-10 anxiety disorders. As shown in Table 3, the $\mathrm{SaD}$ group revealed a somewhat higher rate of comorbid anxiety disorders $(48 \%)$ compared with the $\mathrm{FEP} / \mathrm{SaD}$ group $(35 \%)$ but not significantly different $\left(\chi_{(1)}^{2}=0.88\right)$. The $\mathrm{FEP} /$ no SaD group 


\begin{tabular}{|c|c|c|}
\hline & FEP/SaD group, mean (s.d.) $(n=20)$ & SaD group, mean (s.d.) $(n=31)$ \\
\hline Social Interaction Anxiety Scale & $47.95(9.8)$ & $54 \quad(15)$ \\
\hline Social Phobia Scale & $40.75(13.7)$ & $46.3(15.9)$ \\
\hline Fear of Negative Evaluation Scale & $41.1(10.8)$ & $50.7(8.3)$ \\
\hline Schedules for Clinical Assessment in Neuropsychiatry, autonomic symptoms & $8.21(4.3)$ & $9.93(3.4)$ \\
\hline Calgary Depression Scale for Schizophrenia & $8.25(6.6)$ & $7.4(4.5)$ \\
\hline
\end{tabular}

Table 3 Anxiety disorder diagnoses in the first-episode psychosis (FEP) and the social anxiety disorder (SaD) groups

\begin{tabular}{|c|c|c|c|c|}
\hline \multirow[b]{2}{*}{ Anxiety disorder } & \multicolumn{3}{|c|}{ First-episode psychosis group, $n$} & \multirow{2}{*}{$\begin{array}{l}\text { Social anxiety disorder group, } n \\
\qquad(n=31)\end{array}$} \\
\hline & Without SaD $(n=60)$ & With $\operatorname{SaD}(n=20)$ & Total $(n=80)$ & \\
\hline F.41 Panic disorder & 4 & 4 & 8 & 3 \\
\hline F.40.00 Agoraphobia (no panic) & 1 & 2 & 3 & 3 \\
\hline F.40.01 Agoraphobia (with panic) & 1 & - & 1 & 5 \\
\hline F.40.2 Specific phobias & - & 1 & 1 & 4 \\
\hline Total, $n$ (\%) & $6(10)$ & 7 (35) & $13(16.2)$ & $15(48.3)$ \\
\hline
\end{tabular}

reported a significantly lower rate of comorbid anxiety disorders compared with the other two groups $\left(\chi_{(1)}^{2}=17.2, P<0.01\right)$.

\section{Relationship between depression and social anxiety disorder}

One of the characteristic features of social anxiety is its common co-occurrence with depression; when this comorbidity is present, social anxiety usually precedes the onset of depression. ${ }^{14,31}$ Given the high rate of post-psychotic depression in psychosis, we were interested to examine the relationship between social anxiety and depression following the onset of psychosis in order to see whether they are overlapping or distinct clinical entities.

Using the threshold for moderate depression (CDSS 5) the rate of depression in the whole first-episode psychosis $(n=80)$ sample was $31 \%$; of this group, $52 \%$ also had social anxiety disorder. Conversely, $68.7 \%$ of the FEP group had no or minimal levels of depression and only $12.7 \%$ had social anxiety. In the FEP/ $\mathrm{SaD}$ group, $65 \%$ were depressed; in the $\mathrm{SaD}$ group, $64.5 \%$ were depressed. It appears therefore, that following the onset of psychosis, social anxiety and depression overlap to a significant extent. Although we cannot establish which one may have developed first, these findings emphasise the comorbidity of social anxiety and depression in both psychosis and non-psychosis groups.

\section{Social anxiety and positive psychotic symptoms}

\section{Positive symptoms of psychosis}

The first analysis sought to determine whether those with firstepisode psychosis and social anxiety disorder experience overall more positive symptoms compared with those in the FEP/no $\mathrm{SaD}$ group. One-way between-participants ANOVAs were used to test for differences in PANSS delusions (P1), hallucinations (P3), grandiosity (P5) and suspiciousness/persecution (P6).

No significant differences were found between the two groups with psychosis (with $v$. without social anxiety) in the overall occurrence of delusions $\left(F_{(1,69)}=0.137\right)$ including delusions of grandiosity $\left(F_{(1,69)}=0.76\right)$ and persecution $\left(F_{(1,69)}=2.24\right)$; similarly, the level of hallucinations did not differ between the two groups $\left(F_{(1,69)}<1\right.$, not significant) (Table 4$)$.

The second analysis sought to investigate specifically the relationship between social anxiety and suspiciousness/ persecution as rated by the positive scale of PANSS in the whole first-episode psychosis sample $(n=80)$. A chi-squared test of independence revealed no significant relationship between the diagnosis of social anxiety disorder and PANSS suspiciousness/ persecution $\left(\chi_{(1)}^{2}=0.8\right)$. The relationship between PANSS suspiciousness/persecution and social anxiety was further investigated within the $\mathrm{FEP} / \mathrm{SaD}$ group by identifying those individuals with clinically significant levels of suspiciousness/ persecution ( $\geqslant 4$ on PANSS suspiciousness/persecution) and those with minimal/mild levels ( $<4$ on PANSS suspiciousness/ persecution). Thirteen individuals from the FEP/SaD group scored $\geqslant 4$ on PANSS suspiciousness/persecution and five scored $<4$ (for two people PANSS scores were not available). A oneway between-participants ANOVA was used to test for differences in SIAS, SPS, B-FNE, CDSS and SCAN autonomic symptoms in the groups above. Results showed no significant differences between individuals in the FEP/SaD group with high $v$. low levels of PANSS suspiciousness/persecution in: SIAS $\left(F_{(1,16)}=1.7\right)$, SPS $\left(F_{(1,16)}=1\right)$, CDSS $\left(F_{(1,16)}=0\right)$, FNE $\left(F_{1,15}=0\right)$, SCAN autonomic symptoms $\left(F_{(1,15)}=0\right)$. These findings suggest that the level of delusional suspiciousness did not affect the level of social anxiety within the $\mathrm{FEP} / \mathrm{SaD}$ group.

\section{Perceived threat from persecutors}

The relationship between social anxiety and the nature of the perceived threat from persecutors was examined using the DoT. Of the 20 individuals in the FEP/SaD group, 9 (45\%) reported that they believed someone was 'intending to harm them' $v$. 7 out of 60 $(11.6 \%)$ in the $\mathrm{FEP} /$ no SaD group $\left(\chi^{2}{ }_{1}=10.4, P<0.01\right)$. Thus

Table 4 Positive symptoms on the Positive and Negative Syndrome Scale (PANSS) in the first-episode psychosis (FEP) group and first-episode psychosis with social anxiety disorder (FEP/SaD) group

\begin{tabular}{|lcc|} 
PANSS & $\begin{array}{c}\text { FEP group, } \\
\text { mean (s.d.) } \\
(n=60)\end{array}$ & $\begin{array}{c}\text { FEP/SaD group, } \\
\text { mean (s.d.) } \\
(n=20)\end{array}$ \\
\hline Delusions (P1) & $4.67(2.0)$ & $4.88(2.9)$ \\
\hline Hallucinations (P3) & $4.41(1.5)$ & $4.33(2.0)$ \\
\hline Grandiosity (P5) & $1.77(1.5)$ & $2.16(1.8)$ \\
\hline Suspiciousness/ Persecution (P6) & $3.67(2.1)$ & $4.55(2.2)$ \\
\hline
\end{tabular}


people with psychosis and social anxiety were more likely to express persecutory threat (as rated on the DoT) than those without social anxiety. However, the degree of conviction in the persecutory beliefs, the perceived power of the persecutor, the controllability and 'awfulness' of the threat and associated distress, was not significantly different (Table 5).

These latter tests are based on small samples; however, inspection of the means revealed that the $\mathrm{FEP} / \mathrm{SaD}$ group reported lower levels of threat. The above groups also shared a similar demographic profile: no differences were observed in age $\left(F_{(1,15)}=0\right.$, not significant $)$, gender $\left(\chi^{2}{ }_{1}=2.3\right.$, not significant $)$ or ethnicity $\left(\chi_{3}^{2}=2.8\right.$, not significant $)$.

\section{Persecutory threat and severity of social anxiety in those} with psychosis

Data on the DoT showed that 9 out of the 20 individuals in the FEP/SaD group expressed persecutory threat at the time of the interview. Although the results did not establish a link between social anxiety and the dimensions of persecution (e.g. delusional conviction, power, etc.), the fact that half of the FEP/SaD group did report persecutory beliefs raises the question whether the severity of social anxiety in this subgroup is linked to the nature and degree of persecutory thinking. For the FEP/SaD subsample a one-way ANOVA comparing those with $v$. without persecutory thinking showed no significant differences in: SIAS $\left(F_{(1,19)}=1.05\right)$, SPS $\quad\left(F_{(1,19)}<1\right)$, B-FNE $\quad\left(F_{(1,18)}=2.38\right)$ or depression (CDSS: $\left.F_{(1,19)}<1\right)$. There were no trends in the means suggesting this comparison was underpowered.

A further correlational analysis within the FEP/SaD subgroup who expressed persecutory beliefs $(n=9)$ was conducted in order to examine the relationship between perceived threat/harm from persecutors and the severity of social anxiety using the variables SIAS, SPS and B-FNE. With the exception of a significant relationship between 'awfulness' of threat (DoT 8) and social phobia (SPS) $(r=0.86, P<0.01)$, the results did not show any significant relationship between social anxiety, avoidance, negative evaluative concerns and persecutory thinking within this subgroup.

Overall, these data suggest that the presence of social anxiety disorder: is not linked to the presence of psychosis symptoms including formal persecutory delusions; is not influenced by the severity of persecutory delusions within the FEP/SaD group; but when asked on the DoT whether they 'know someone is trying to harm them' nearly half of the FEP/SaD group agreed ( $v .11 \%$ of those without social anxiety disorder). However, the level of social anxiety in this subgroup was not influenced by the level of persecutory beliefs.

\section{Autonomic symptoms of anxiety and positive psychotic symptoms}

Previous research has reported a relationship between general anxiety and positive symptoms of psychosis. Freeman et al, ${ }^{17}$

\begin{tabular}{|c|c|c|}
\hline Dot & $\begin{array}{l}\text { FEP group, } \\
\text { mean (s.d.) } \\
\quad(n=7)\end{array}$ & $\begin{array}{c}\text { FEP/SaD group, } \\
\text { mean (s.d.) } \\
(n=9)\end{array}$ \\
\hline Power of persecutor & $9.3(1.2)$ & $7.4(2.5)$ \\
\hline Delusional conviction & $97.1(4.8)$ & $90(19.3)$ \\
\hline Delusional distress & $9.4(0.8)$ & $8.1(2.7)$ \\
\hline Awfulness of threat & $9.3(1.2)$ & $9.1(1.4)$ \\
\hline Controllability & $2.4(3)$ & $3.4(3.8)$ \\
\hline
\end{tabular}

suggest that anxiety is likely to play an important role in the formation of persecutory delusions since their content, that is anticipation of threat and danger, reflects the thematic content of anxiety disorders. We attempted to verify this link in the whole FEP group $(n=80)$ by examining the relationship between SCAN autonomic symptoms of anxiety and the PANSS positive symptoms scales.

Correlations between the total number of SCAN autonomic anxiety symptoms and delusions (P1), hallucinations (P3), grandiosity (P5) and suspiciousness/persecution (P6), revealed a significant relationship with hallucinatory behaviour (P3) $(r=0.27, P<0.05)$ and suspiciousness/persecution (P6) $(r=0.25$, $P<0.05)$. These data show a correlation between psychosis symptoms and general anxiety confirming previous findings.

\section{Discussion}

\section{Prevalence of social anxiety in psychosis}

The findings of this study indicate that the rate of ICD-10 social anxiety disorder in a representative sample of 80 people with firstepisode psychosis is $25 \%$. Similar rates have been reported in previous studies with enduring schizophrenia. ${ }^{10,13,32}$ Tibbo et $a l^{32}$ reported a rate of $23.3 \%$ in 30 out-patients with chronic schizophrenia. Pallanti et al ${ }^{10}$ reported a rate of $36.3 \%$ in a similar group, and in Birchwood et al's study ${ }^{11}$ social anxiety was diagnosed in $23(29 \%)$ out of 79 people with first-episode psychosis.

In addition to the $25 \%$ with formal social anxiety disorder, there was also a further $11.6 \%$ who reported clear social interaction difficulties and/or signs of avoidance not sufficient though to reach formal diagnostic criteria. These 'borderline' individuals, although not satisfying formal criteria, were nevertheless reporting interpersonal difficulty that may well warrant intervention at a clinical level. In addition, individuals with subsydromal levels of social anxiety and social interaction difficulties were also present. The importance of not excluding cases of mild-moderate severity from diagnostic manuals has been stressed by Kessler et al. ${ }^{33}$ The authors reported how DSM-III-R disorders in a nationally representative sample (National Comorbidity Survey) varied in severity from severe to serious, moderate and mild levels. All these case categories, including mild cases, at baseline were significantly related to later clinical outcomes such as hospitalisation, suicide attempts and work disability (in a dose-response fashion). The authors argued that mental illness can be placed on a severity gradient and that mild cases should not be ignored; rather the decision to be treated should be based on cost-effectiveness in an attempt to prevent transition to serious disorders.

In our sample of people with first-episode psychosis $(n=80)$, social anxiety appears to be distributed on a continuum of severity with mild or subsyndromal levels at one end moving to moderate levels of severity (borderline cases) and ending at the opposite pole with social anxiety disorder.

\section{Social anxiety in psychosis v. non-psychosis groups}

Overall there were few differences between the FEP and SaD groups in the quality and quantity of their social anxiety. Both groups reported similar levels of social anxiety and social avoidance; the number and severity of autonomic anxiety symptoms was comparable. Intense apprehension about being criticised and negatively evaluated during social encounters was prominent in both groups, although somewhat higher in the $\mathrm{SaD}$ group. Findings also revealed that social anxiety in both $\mathrm{SaD}$ and $\mathrm{FEP} / \mathrm{SaD}$ groups occurred in the context of an equally 
high level of other anxiety disorders underlying the similarity of the two groups.

The overall profile in both groups is further characterised by marked levels of depressed mood in $64.5 \%$ of the SaD and $65 \%$ of the FEP/SaD group, confirming previously reported findings of the substantial rates of depression in people without psychosis ${ }^{14}$ and those with psychosis ${ }^{34,35}$ with social anxiety. Social anxiety and depression have consistently been linked in the literature both phenomenologically and developmentally. ${ }^{14,36}$ Both disorders have an early onset, usually in childhood or mid-teenage years $^{14,37,38}$ and tend to show continuity into adulthood. The interrelationship of social anxiety and depression was also found in this sample of young people with psychosis and in our $\mathrm{SaD}$ group. Post-psychotic depression in the FEP sample was 31\% at cross-section, in line with previous studies, ${ }^{39}$ and in approximately $50 \%$ of these people this overlapped with social anxiety disorder. We suggest therefore that social anxiety and depression are not distinct clinical entities in psychosis, as Pallanti et a $l^{15}$ have suggested, rather that they appear to overlap phenomenologically, they share similar course patterns in relation to psychosis and they are both independent of positive symptoms (no relationship was found in the FEP/SaD group between the total CDSS score and PANSS positive symptoms ( $P$ not significant $)$ ). However, we have no evidence from the present cross-sectional study whether the development sequence observed by Stein et $a l^{14}$ is present in psychosis.

Overall, we conclude that the phenomenology and severity of social anxiety in psychosis is indistinguishable from non-psychosis social anxiety disorder. Furthermore, the findings emphasise the comorbidity of social anxiety and depression in both groups.

\section{Social anxiety and positive psychosis symptoms}

A key aim of this study was to examine whether social anxiety in psychosis is driven by the presence of psychosis symptoms, especially paranoia and persecutory delusions. The findings were not quite as anticipated. First, we found no differences in the positive symptoms of the PANSS between individuals with psychosis and with $v$. without social anxiety; including no relationship between suspiciousness/persecution as rated on the PANSS and social anxiety in the whole FEP sample. Furthermore, the level of PANSS suspiciousness/persecution did not affect the severity of social anxiety within the FEP/SaD group.

However, when participants were asked on the DoT whether they believed someone was 'intending to harm them', a substantially greater percentage of socially anxious $v$. non-socially anxious individuals with psychosis $(45 \% \quad v .11 .6 \%)$ replied affirmatively, although no differences were observed in the dimensions and quality of the perceived threat (e.g. power of persecutor, delusional conviction, distress) between the two groups; and no link between social anxiety and dimensions of persecutory threat within the subgroup of those with social anxiety disorder who expressed persecutory beliefs (DoT). The last line of evidence is of particular interest since it suggests that even among those in the FEP/SaD group, social anxiety is not necessarily contaminated by ongoing persecutory beliefs. This proposal makes clinical sense since social anxiety is generalised across all people and social encounters, whereas paranoia is often, but not always, focused on particular individuals or groups (e.g. certain neighbours, old school friends, mafia, etc).

The above findings then suggest that in first-episode psychosis, social anxiety is not simply an epiphenomenon of clinical paranoia and has more than one causal pathway. For a subgroup $(45 \%)$ of socially anxious people with psychosis however, persecutory threat and anticipated harm were present (but not measurable on the PANSS) and it is therefore important to understand and delineate the processes that underlie the relationship between social anxiety and persecutory threat in this subsample. We propose three pathways:

(a) social anxiety pre-dates the onset of persecutory beliefs and serves to trigger and/or maintain persecutory thinking in this subgroup;

(b) social anxiety and persecutory thinking develop concurrently in the early phase of psychosis and follow a similar course;

(c) social anxiety develops in some as a consequence of paranoid thinking.

The first pathway suggests the possibility of individuals developing social anxiety, avoidance and withdrawal in the prodrome or earlier phase of psychosis. Paranoid beliefs and ideas of persecution emerge later on and, as Freeman et a $l^{40,41}$ has suggested, build upon already present social anxieties such as fear of rejection which help sustain and perhaps reinforce paranoid fears.

The second pathway suggests that social anxiety and persecutory ideation develop at the same time and follow a similar course. The two concepts are intertwined and the underlying fears are almost indistinguishable. It is expected that in these individuals addressing and treating paranoid fears would result in a drop of social anxiety. However, this would not account for the $55 \%$ who have social anxiety disorder without concurrent persecutory beliefs.

Finally, the third pathway identifies social anxiety as a by-product of persecutory beliefs. Here the beliefs about the persecutory intent of individuals or groups of individuals generalise to everyone in the individual's social world, as a safety behaviour for example.

For the $55 \%$ of the $\mathrm{FEP} / \mathrm{SaD}$ group who did not have concurrent paranoia, we may need to invoke different mechanisms. In this study we were not able to plot reliably the time course of social anxiety in the FEP group; however, at least $12 / 20$ of the FEP/SaD group reported their social anxiety problems to have become prominent following the onset of psychosis. This suggests that social anxiety emerged de novo in this group or built on low-level interpersonal anxiety, consistent with our previous findings. ${ }^{11}$

Although we were not able to reliably assess and establish the presence of premorbid social anxiety, we propose that subsyndromal levels of social anxiety (e.g. interpersonal sensitivity or situational anxiety) do form part of the prodrome in people who develop psychosis. 'Situational' anxiety was found to be common in a high-risk sample and among the best predictors of transition to psychosis in the Edinburgh High Risk study. ${ }^{4,16}$ These 'endophenotypes' in the context of known social risk factors for psychosis (e.g. abuse, parental loss) ${ }^{42,43}$ may interact and lead to the development of social anxiety disorder before and after the onset of psychosis. In individuals with social anxiety disorder without psychosis, current theory argues for a similar interaction of 'trait' disposition in the context of adverse social risk factors. ${ }^{14,44}$

Links with general anxiety symptoms and positive psychosis symptoms, specifically delusions, were reported, in line with Freeman et $a l^{17}$ who argue that anxiety forms an integral part of the psychotic symptomatology which is likely to play an important role in the development and maintenance of persecutory delusions, due to similar themes and processes. Social anxiety, however, has a somewhat different quality and its presence in psychosis has a complex relationship with persecutory thinking. They may share however common pathways and developmental risk factors known to operate in psychosis and social anxiety (e.g. early traumatic experiences, dysfunctional 
parental bonding $)^{42,43}$ that may create a vulnerability for the emergence of emotional problems during adolescence and into psychosis. ${ }^{44}$ We investigate this possibility in a forthcoming paper.

\section{Methodological issues and limitations}

This is the first study to undertake a detailed phenomenological analysis of social anxiety and to compare it with a group without psychosis. Although we established that social anxiety is not linked to positive symptoms and particularly paranoia, we cannot rule out the possibility that social interaction difficulties and avoidance developed as a by-product of alogia, anedonia, blunted affect and other negative symptoms. However, neither Pallanti et al ${ }^{15}$ nor our previous research ${ }^{11}$ found any link between social anxiety and negative symptoms. Our measure of autonomic symptoms of anxiety used the list of autonomic symptoms in the SCAN, section 4 , and these were summed to obtain the total number. Although we did not use a standard questionnaire, this list of symptoms is used to establish the various anxiety diagnoses and was shown to be reliable (Cronbach's alpha was 0.91 ).

The SCAN was used in order to measure premorbid levels of social anxiety in the FEP/SaD group. Given the retrospective nature of these data there is a risk of recall bias; although 12/20 of the $\mathrm{FEP} / \mathrm{SaD}$ group reported that their social anxiety difficulties developed after they have been diagnosed with psychosis, it is possible that low-level anxiety symptoms were present before the onset of psychosis and these became clinically prominent following the first episode. In a study by Birchwood et l $^{11}$ no differences were observed in overall premorbid interpersonal functioning, suggesting that social anxiety disorder developed after the psychosis onset in a substantial proportion of the sample. We also note that some differences in the demographic characteristics of the samples were present. Both the $\mathrm{SaD}$ and FEP/SaD groups showed a female excess, whereas in the $\mathrm{FEP} /$ no $\mathrm{SaD}$ group an expected male excess was observed.

\section{Implications}

Social anxiety is a prevalent affective disorder present in the early phase of psychosis and appears to become clinically prominent after its onset. There was no evidence that social anxiety is simply a 'phenocopy' arising from psychotic symptoms, particularly paranoia and accordingly warrants a distinct research and clinical focus. The processes and themes that underlie social anxiety preand post-psychosis warrant further investigation.

Maria Michail, MSc, PhD, Max Birchwood, Phd, DSC FBPSS, School Psychology, University of Birmingham, Edgbaston, Youth Services Programme, Birmingham and Solihull Mental Health Foundation Trust, UK

Correspondence: Maria Michail, School of Psychology, University of Birmingham, Edgbaston, Birmingham B15 2TT, UK. Email: m.michail@bham.ac.uk

First received 28 Mar 2008, final revision 18 Feb 2009, accepted 3 Mar 2009

\section{References}

1 Murray V McKee I, Miller PM, Young D, Muir WJ, Pelosi AJ, et al. Dimensions and classes of psychosis in a population cohort: a four-class, four-dimension model of schizophrenia and affective psychoses. Psychol Med 2005; 35: 499-510.

2 Koreen A, Siris S, Chakos M, Alvir J, Mayerhoff D, Lieberman J. Depression in first-episode schizophrenia. Am J Psychol 1993; 150: 1643-8.

3 Hafner $\mathrm{H}$, Loffler W, Mauer K, Hambrecht M, an der Heiden W. Depression, negative symptoms, social stagnation and social decline in the early course of schizophrenia. Acta Psychiatr Scand 1999; 100: 105-18.
4 Cunningham Owens DG, Miller P, Lawrie SM, Johnstone EC. Pathogenesis of schizophrenia: a psychopathological perspective. Br J Psychiatry 2005; 186: 386-93.

5 McGlashan T, Carpenter W. An investigation of the post-psychotic depressive symptom. Am J Psychol 1976; 133: 14-9.

6 Birchwood M, Iqbal Z, Chadwick P, Trower P. Cognitive approach to depression and suicidal thinking in psychosis: I. Ontogeny of post-psychotic depression. Br J Psychiatry 2000; 177: 516-28.

7 Davidson J, Hughes D, George L, Blazer D. The epidemiology of social phobia: findings from the Duke Epidemiological Catchment Area Study. Psychol Med 1993; 23: 709-18.

8 Cassano GB, Pini S, Saettoni M, Dell'Oso L. Multiple anxiety disorder comorbidity in patients with mood spectrum disorders with psychotic features. Am J Psychol 1999; 156: 474-6.

9 Goodwin DR, Amador FX, Malaspina D, Yale AS, Goetz RR, Gorman MJ. Anxiety and substance use comorbidity among inpatients with schizophrenia. Schizophr Res 2003; 61: 89-95.

10 Pallanti S, Quercioli L, Hollander E. Social anxiety in outpatients with schizophrenia. A relevant cause of disability. Am J Psychol 2004; 161: 53-8.

11 Birchwood M, Trower P, Brunet K, Gilbert P, Iqbal Z, Jackson C. Social anxiety and the shame of psychosis: a study in first-episode psychosis. Behav Res Ther 2007; 45: 1025-37.

12 Penn DL, Hope DA, Spaulding W, Kucera J. Social anxiety in schizophrenia. Schizophr Res 1994; 11: 277-84.

13 Cossof J, Hafner R. The prevalence of comorbid anxiety in schizophrenia, schizoaffective disorder and bipolar disorder. Aust N Z J Psychiatry 1998; 32: 67-72.

14 Stein $\mathrm{M}$, Fuetsch $\mathrm{M}$, Muller $\mathrm{N}$, Hofler $\mathrm{M}$, Lieb $\mathrm{R}$, Wittchen $\mathrm{H}$. Social anxiety and the risk of depression. Arch Gen Psychiatry 2001; 58: 251-6.

15 Pallanti S, Quercioli L, Hollander E. Dr Pallanti and colleague reply (letter). Am J Psychiatry 2005; 162: 400-1.

16 Johnstone $C E$, Ebmeier KP, Miller P, Owens DGC, Lawrie SM. Predicting schizophrenia: findings from the Edinburgh High-Risk Study. Br J Psychiatry 2005; 186: 18-25.

17 Freeman D Garety PA, Kuipers E. Persecutory delusions: developing the understanding of belief maintenance and emotional distress. Psychol Med 2001; 31: 1293-306.

18 World Health Organization. Schedules for Clinical Assessment in Neuropsychiatry (version 2.1). WHO, 1999.

19 Kay SR, Fiszbein A, Opler LA. The positive and negative syndrome scale (PANSS) for schizophrenia. Schizophr Bull 1987; 13: 261-76.

20 World Health Organization. The ICD-10 Classification of Mental and Behavioural Disorders. WHO, 1993.

21 Mattick RP, Clarke JC. Development and validation of measures of social phobia and scrutiny fear and social interaction anxiety. Behav Res Ther 1998; 36: $455-70$.

22 Peters L. Discriminant validity of the Social Phobia and Anxiety Inventory (SPAI), the Social Phobia Scale (SPS) and the Social Interaction Anxiety Scale (SIAS). Behav Res Ther 2000; 38: 943-50.

23 Leary MR. A brief version of the Fear of Negative Evaluation Scale. Pers SoC Psychol Bull 1983; 9: 371-5.

24 Cox BJ, Swinson RP. Assessment and measurement. In Social Phobia: Clinical and Research Perspective (ed MB Stein): 261-91. American Psychiatric Press, 1995

25 Freeman D, Garety P, Kuipers E. Persecutory delusions: developing the understanding of belief maintenance and emotional distress. Psychol Med 2001; 31: 1293-306.

26 Addington D, Addington J, Maticka-Tyndale E. Assessing depression in schizophrenia: the Calgary Depression Scale. Br J Psychiatry 1993; 163 (suppl 22): 39-44.

27 Beck AT, ward $\mathrm{CH}$, Mendelson M, Mock J, Erbaugh, J. An inventory for measuring depression. Arch Gen Psychiatry 1961; 5: 561-71.

28 Trower P, Birchwood M, Meaden A, Byrne S, Nelson A, Ross K. Cognitive therapy for command hallucinations: randomised controlled trial. $\mathrm{Br} \mathrm{J}$ Psychiatry 2004; 184: 312-20.

29 Muller MJ, Brening H, Gensch C, Klinga J, Kienzle B, Müller K. The Calgary Depression Rating Scale for schizophrenia in a healthy control group: psychometric properties and reference values. J Affect Disord 2005; 88: 69-74.

30 American Psychiatric Association. Diagnostic and Statistical Manual of Mental Disorder (4th edn) (DSM-IV). APA, 1994.

31 Stein, M., Chavira, D. Subtypes of social phobia and comorbidity with depression and other anxiety disorders. J Affect Disord 1998; 50 (suppl); s11-162. 
32 Tibbo $\mathrm{P}$, Swainson J, Chue $\mathrm{P}$, LeMelledo J-M. Prevalence and relationship to delusions and hallucinations of anxiety disorders in schizophrenia. Depress Anxiety 2003; 17: 65-72.

33 Kessler RC, Merikangas KR, Berglund P, Eaton WW, Koretz SD, Walters EE. Mild disorders should not be excluded from the DSM-V. Arch Gen Psychiatry 2003, 60: 1117-22

34 Kingsep $P$, Nathan $P$, Castle D. Cognitive behavioural group treatment for social anxiety in schizophrenia. Schizophr Res 2003; 63: 121-9.

35 Gumley A, O'Grady M, Power K, Schwannauer M. Negative beliefs about self and illness: a comparison of individuals with psychosis with and without comorbid social anxiety disorder. Aust N Z J Psychiatry 2004; 38: 960-4.

36 Stein $M$, Tancer $M$, Gelernter $C$, Vittone $B$, Uhde T. Major depression in patients with social phobia. Am J Psychiatry 1990; 147: 637-9.

37 Schneier F, Johnson J, Horing C, Liebowitz A, Weissman M. Social phobia: comorbidity and morbidity in an epidemiological sample. Arch Gen Psychiatry 1992; 49: 282-8.

38 Wittchen $\mathrm{H}-\mathrm{U}$, Stein $\mathrm{M}$, Kessler R. Social fears and social phobia in a community sample of adolescents and young adults: prevalence, risk factors and comorbidity. Psychol Med 1999; 29: 309-23.
39 Siris SG. Depression and schizophrenia. In Schizophrenia (eds SR Hirsch, DR Weinberger): 128-45. Blackwell, 1995.

40 Freeman D, Garety PA, Bebbington P, Slater M, Kuipers E, Fowler D, et al The psychology of persecutory ideation. II: a virtual reality experimental study. J Nerv Ment Dis 2005; 193: 309-15.

41 Freeman $D$, Garety $P A$, Bebbington $P E$, Smith $B$, Rollinson R, Fowler $D$, et al. Psychological investigation of the structure of paranoia in a non-clinical population. Br J Psychiatry 2005; 186: 427-35.

42 Kirkbride JB, Morgan C, Fearon P, Dazzan P, Murray RM, Jones PB. Neighbourhood-level effects on psychoses: re-examining the role of context. Psychol Med 2007; 37: 1413-25.

43 Morgan C, Kirkbride J, Leff J, Craig T, Hutchinson G, McKenzie K, et al. Parental separation, loss and psychosis in different ethnic groups: a casecontrol study. Psychol Med 2007; 37: 495-503.

44 de Wilde A, Rapee RM. Do controlling maternal behaviours increase state anxiety in children's responses to a social threat? A pilot study. J Behav Ther Exp Psychiatry 2008; 39: 526-37.

\section{Hope to live}

Gethin Morgan

Hope to continue living is rarely irrevocably lost. Even in mental illness and depths of despair hope is still there waiting to return. In chronic physical disability hope and comfort can be regained through seemingly trivial symptomatic improvement. It can survive in apparently hopeless situations. So surely we should fan whatever embers of hope remain, and never confirm despair. To begin, just listen with realistic optimism for as long as it takes. It soon becomes clear that such hope can indeed spring eternal in the human breast; and from it flows a renewed conviction that life is after all worthwhile. 